Neurosurg Focus 12 (4):Article 4, 2002, Click here to return to Table of Contents

\title{
Factors affecting profitability for craniotomy
}

\author{
A. John Popp, M.D., Todd Scrime, M.T. (ASCP), Benjamin R. Cohen, M.D., \\ Paul J. Feustel, Ph.D., Karen Petronis, R.N., Sharon Habiniak, R.N., \\ John B. Waldman, M.D., and Margaret M. Vosburgh, R.N., M.S., M.B.A. \\ Division of Neurosurgery, Albany Medical College; and Patient Care Services/Surgical and \\ Interventional Service, Department of Quality Management, and Department of Center Operations, \\ Albany Medical Center, Albany, New York
}

\begin{abstract}
Object. The authors studied factors influencing hospital profitability after craniotomy in patients who underwent craniotomy coded as diagnosis-related group (DRG) 1 (17 years of age with nontraumatic disease without complication) and who met their hospital's craniotomy pathway criteria and had a hospital length of stay 4 days or less during a 20-month period.

Methods. Data in all patients meeting these criteria (76 cases) were collected and collated from various hospital databases. Twenty-one cases were profitable and 55 were not. Variables traditionally influencing cost of care, such as surgeon, procedure, length of operation, and pharmacy use had no significant effect on whether a patient was profitable. The most important influence on profitability was the individual payor. Cases in which care was reimbursed under the prospective payment system based on DRGs were nearly always profitable whereas those covered by per diem plans were nearly always nonprofitable.

Conclusions. 1) Hospital information systems should be customized to deliver consolidated data for timely analysis of cost of care for individual patients. This information may be useful in negotiating profitable contracts. 2) A clinical pathway was successful in reducing the difference in cost of care between profitable and nonprofitable postcraniotomy cases. 3) In today's health care environment both cost containment and revenue assume importance in determining profitability.
\end{abstract}

KEY WORDS • craniotomy • cost analysis • revenue - managed care

Hospital administrators are being forced to scrutinize carefully their revenues and expenses because of the current medicoeconomic environment. ${ }^{3,6}$ Hospital officials have implemented various strategies to reduce the cost of care by using CQI and clinical pathways. ${ }^{7}$ The complexity of revenue streams and internal expense allocation, however, make accurate financial analyses problematic. Because our institution has a successful program involving clinical pathway development and strong neurosurgeon participation in our CQI process, we sought to determine what factors were involved in profitability in cases classified in a single federal DRG (DRG 1): patients greater than 17 years of age with nontraumatic disease in whom craniotomy is performed without complication.

Abbreviations used in this paper: $\mathrm{AMC}=$ Albany Medical Center; $\mathrm{CI}=$ confidence interval; $\mathrm{CQI}=$ continuous quality improvement; DRG = diagnosis-related group; HIS = hospital information system; LOS = length of hospital stay; OR = odds ratio; PPS = prospective payment system.

\section{CLINICAL MATERIAL AND METHODS}

\section{Data Collection}

Institution review board approval for data analysis and publication was obtained. The majority of the data were extracted from two sources: HIS software and cost-accounting reporting software. The demographic data generated by HIS were reported from a system developed by QuadraMed Corporation (SanRafael, CA), whereas the cost-accounting data from the finance department were generated using a reporting system developed by Transition Systems, Inc. (TSI), which has since been acquired by Eclipsys (Boca Raton, FL). All of the operating room times were taken from the operating room database SurgiServe (Roseville, MN).

At AMC we do not use a central integrated clinical database or electronic medical records (both in development). Thus, to link disparate data elements from multiple departments, many ad hoc databases have been developed to create relationships among datasets that otherwise 
could not be made. The data provided for this analysis were generated monthly from HIS and the finance department and sent to the database located in the quality management department (Table 1). All individually identifiable patient information subsequently was stripped from the datasets. This study includes all cases coded as Federal DRG 1 in which the patients were discharged between January 1, 2000, and August 31, 2001.

\section{Patient Population}

The inclusion criteria were designed to allow for the most homogeneous population possible without yeilding too few cases for the study to be valid. Certain criteria were imposed to define the study population. Patients included were those in whom the discharge diagnosis was compatible with DRG 1, to ensure a similar magnitude of surgery among patients and hence a similar utilization of resources. This selection was performed using a "grouper" that automatically compiles the correct mixture of DRG 1-related diagnoses and/or procedures. The entrance criteria were further narrowed to include only DRG 1 cases that met our hospital's craniotomy pathway criteria (Table 2). Finally, to remove the impact of outliers, only patients for whom the LOS was 4 days or less were included.

The following diagnoses are examples of those fitting this description: malignant brain tumor, meningioma, unruptured aneurysm, and acoustic neuroma.

\section{Data Analysis}

Once the patient population was defined, we sought to determine the factors that influenced the profitability of an individual case by using the following formula: profit $=$ net revenue - actual total cost. Net revenue includes reimbursement from payors and from Graduate Medical Education reimbursement; actual total cost represents the sum of total fixed cost and total variable cost. The cost-

TABLE 1

Data elements collected from the HIS and finance department at $A M C^{*}$

Individual Patient Identifier

HIS

medical record no.

admission date

discharge date

total LOS

age

sex

admission source

discharge disposition

attending physician

primary, secondary, tertiary diagnoses ICD-9-CM code primary, secondary, tertiary procedures ICD-9-CM code

finance department

primary payor

actual total cost

actual fixed cost

actual total variable cost

net revenue

profit (net revenue - actual total cost)

* ICD-9-CM = International Classification of Diseases, 9th revision, Clinical Modification.
TABLE 2

Diagnosis and procedure codes that exclude DRG 1 cases from craniotomy pathway

\begin{tabular}{cl}
\hline $\begin{array}{c}\text { ICD-CM9 Code } \\
\text { Pathway Exclusions }\end{array}$ & \multicolumn{1}{c}{ Description } \\
\hline diagnosis & \\
430 & subarachnoid hemorrhage \\
431 & intracerebral hemorrhage \\
procedure & incision \& drainage of cranial sinus \\
01.21 & hemispherectomy \\
01.52 & ventriculostomy \\
02.2 & ventricular shunt to abdominal cavity \& organs \\
02.34 & replacement of ventricular shunt \\
02.42 & removal of ventricular shunt \\
02.43 & implantation of intracranial neurostimulator \\
02.93 & insertion or replacement of skull tongs or halo \\
02.94 & traction device \\
92.3 & stereotactic radiosurgery \\
\&/or 96.04 & reinsertion of endotracheal tube \\
\hline
\end{tabular}

accounting system allocates "actual total cost" through the use of relative value units. Total variable cost includes variable labor, variable supply, and variable other. Total fixed cost is comprised of fixed labor, fixed equipment, fixed other, and fixed indirect.

The Eclypsis system is set up to allocate cost into different cost centers including, for example, the operating room, pharmacy, vascular radiology, anesthesia, physical medicine and rehabilitation, hematology, and room and board. The room and board charges include supply costs averaged biannually, whereas other areas such as pharmacy and operating room post specific charges to patients.

Graduate Medical Education reimbursement is averaged across cases that would have benefited from those funds in particular hospital areas or services, thus affecting the net revenue side of the aforementioned equation. In the Eclypsis system net revenue is modeled based on historical data and contract adjustments. The use of modeled net revenue was verified by comparing actual total reimbursement for each individual. Because Graduate Medical Education reimbursement is not part of the actual total reimbursement, this factor was excluded from the modeled net revenue. Because no individual case categorized as nonprofitable ever became profitable whether the actual total reimbursement or the modeled revenue was used in the formula, we used the modeled revenue for all subsequent determinations of profitability. The payor's method of payment, whether by PPS based on DRGs or by per diem payment was determined.

Logistic regression analysis was used to assess the influence of potential predictor variables on profitability. Profitability was dichotomized; each case was classified as either profitable or nonprofitable. The OR of nonprofitability was computed for each potential predictor variable separately and is reported with its 95\% CI. Potential predictor variables tested were patient age, operating room time, American Society of Anesthesiologists class, whether the patient was discharged to a rehabilitation facility, LOS, surgeon, procedure, total cost, and payor. When appropriate, intergroup profit differences were assessed using the nonparametric Mann-Whitney U test 
for two groups and the Kruskal-Wallis analysis of variance for more than two groups. Significance was accepted at the p less than 0.05 level; we used Statistica 6.0 software (StatSoft, Tulsa, OK).

\section{RESULTS}

The number of cases coded as DRG 1 during the study period was 284; of the 96 patients eligible for the DRG 1 pathway, an LOS of 4 days or fewer was documented in 76. Of the 76 cases of craniotomy analyzed, 21 were profitable and 55 were not. Total cost analysis conducted by individual case was complicated by varying resource utilization, including, nurse/patient ratio and operating room time. Figure 1 shows the breakdown of total cost for the study population in our hospital's cost accounting system. The operating room, room and board, pharmacy, and anesthesiology were the four "cost centers" that accounted for greater than $80 \%$ of the total cost of care. We analyzed the difference in mean total cost per case between the profitable and nonprofitable cases among all cost centers. Only five of 25 cost centers showed a difference of greater than or equal to $\$ 100$ in mean total cost between profitable and nonprofitable cases. In the two cost centers - angiography and clinical neurophysiological monitoring - there were too few cases in each group for the difference to be statistically significant. There was no statistically significant difference between operating room, blood bank, or pharmacy costs in nonprofitable compared with profitable cases. Paradoxically, the mean cost per case of room and board was higher in the profitable rather than nonprofitable cases ( $\$ 2207$ and $\$ 1991$, respectively).

There was no statistically significant association between nonprofitability and patient age, operating room time, American Society of Anesthesiologists class, whether the patient was discharged to a rehabilitation facility, LOS, surgeon, procedure, or total cost (Table 3). The odds of nonprofitability depended significantly upon the payor (Table 3); for example, the odds of nonprofitability for the

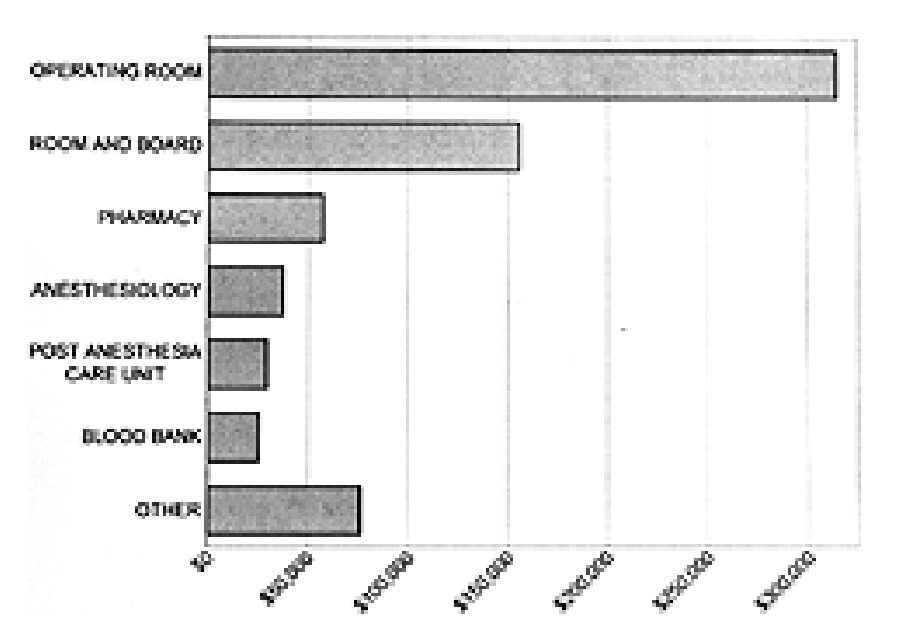

Fig. 1. Bar graph demonstrating breakdown of total cost for 76 craniotomies by cost center. The highest six cost centers are shown. The $\mathrm{x}$-axis shows the cost (dollars) and the $\mathrm{y}$-axis the cost center. The operating room, room and board, pharmacy, and anesthesiology accounted for more than $80 \%$ of the total cost of care.
TABLE 3

Odds ratio for cases of nonprofitability

\begin{tabular}{lccc}
\hline \hline & $\begin{array}{c}\text { No. of } \\
\text { Cases }\end{array}$ & OR & $95 \%$ CI \\
& 76 & & \\
\hline total no. of patients & & \\
age (OR/yr of age) & & 0.97 & $0.93-1.01$ \\
operating room time (OR/hour) & & 1.12 & $0.90-1.41$ \\
ASA class (OR/unit increase) & & 1.13 & $0.40-3.17$ \\
LOS (OR/day stay) & 9 & 0.70 & $0.34-1.43$ \\
discharge other than home & $9.16-3.33$ \\
surgeon (OR relative to all others combined) & & \\
$\quad$ surgeon 1 & 25 & 0.97 & $0.33-2.88$ \\
surgeon 2 & 17 & 2.05 & $0.51-8.20$ \\
surgeon 3 & 14 & 1.50 & $0.37-6.16$ \\
surgeon 4 & 9 & 0.43 & $0.10-1.81$ \\
procedure (OR relative to all others combined) & & \\
malignant tumor & 19 & 1.09 & $0.33-3.60$ \\
meningioma & 18 & 0.99 & $0.30-3.28$ \\
vascular & 9 & 1.39 & $0.26-7.48$ \\
payor (OR relative to all others combined) & & \\
payor 1 & 26 & 16.7 & $2.0-138$ \\
payor 2 & 14 & high* & \\
payor 3 & 9 & 0.30 & $0.01-0.27$ \\
payor 4 & 8 & high* & \\
payor 5 & 1.57 & $0.16-15.5$ \\
payor 6 & 5 & 1.57 & \\
\hline
\end{tabular}

* Because all cases were nonprofitable, no OR were estimated.

$\$$ All cases were profitable.

payor with the most cases (Payor 1, 26 cases [Table 3]) was 16 -fold $(95 \%$ CI $2-138)$ that for all other payors combined. In addition, the actual profit was grouped according to surgeon (Fig. 2), diagnosis (Fig. 3), and payor (Fig. 4). Profit was found to be different only when grouped by payor (Kruskal-Wallis analysis of variance, $\mathrm{p}<0.01$ ). Among payors with more than five cases, distinctly nonprofitable and profitable payors emerged, with the higher-volume payors tending to be less profitable (Fig. 4). Furthermore, 54 of 55 nonprofitable cases had payors (Payors 1, 2, 4, 5, and others) who reimbursed by per diem payments.

\section{DISCUSSION}

\section{Health Care Environment}

The dual impact of managed care and declining federal and state hospital reimbursements has forced hospital administrators to scrutinize carefully their fundamental approach to the "business" of medicine if they wish to remain solvent. Cost-subsidizing/shifting strategies, which once allowed subsidization of under- and unfunded programs by highly profitable hospital services, are no longer viable because of the shrinkage of the profit margins in programs that were once "cash cows" for hospitals. Many hospital officials responded to these changing paradigms by improving efficiency of case "throughput" while maintaining quality of care by using clinical pathways structured by CQI principles. ${ }^{1}$ Clinical pathways were used to standardize care in cases of patients in which there were similar problems to decrease the influence of rework and physicians variability on LOS and cost of care. To keep pace with technological and medical advancements, clinical pathways have evolved into a dynamic plan in which 
A. J. Popp, et al.

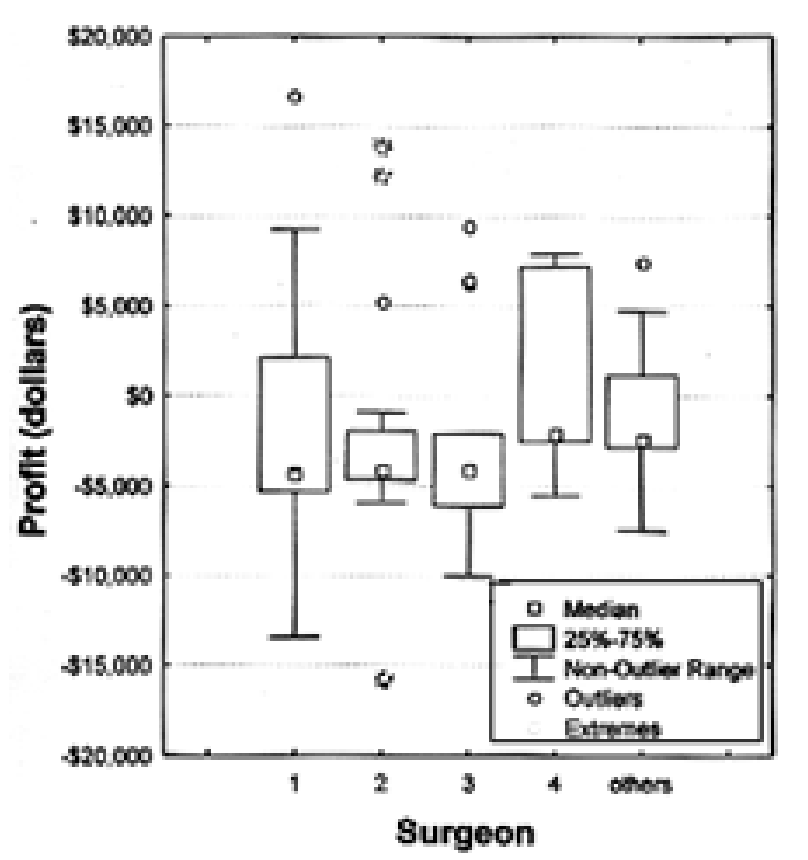

Fig. 2. Box plot of profit categorized by surgeon demonstrating no difference in profit among surgeons (Kruskal-Wallis test, $\mathrm{p}=0.29$ ). Surgeons are in order of number of cases treated, with Surgeon 1, 2, 3 and 4 performing $25,17,14$, and nine craniotomies, respectively. Two other surgeons performed a total of 11 craniotomies.

ongoing revision is required. Quality of care in the setting of reduced LOS and cost was monitored and enhanced by continuous evaluation of parameters linked to outcome such as patient satisfaction and incidence of complications. The result of these tactics on hospitals has been improved efficiency in the delivery of quality care at less cost. ${ }^{5,8}$

Although this internal response within hospitals seems well conceived and appropriate to minimize a perceived inefficiency, the other component of the cost/revenue formula - that is, revenue - is equally important in determining profitability. This may seem self-evident in concept; however, it is surprisingly difficult to ascertain whether similar cases can each be managed profitably and how a hospital might alter its delivery of care to change a nonprofitable product line into a profitable one. The complexity of the medical "product," in the diversity of services necessary to care for the spectrum of potential cases that may require hospital care, is one component of this difficulty. Simply, hospitals are not manufacturing a single product; rather, they are required to be prepared continuously to manage, medically and/or surgically, thousands of disorders in patients with varying ages, physiologies, and severities of illness.

This complex internal environment is matched by an equally complex external reimbursement environment-a welter of governmental and private payors that have multiple payment plans, some using a PPS based on DRGs and others on contracted per diem rates. An individual inclined to "game the system" might be tempted to keep patients with a per diem reimbursement plan in the hospital longer than medically indicated. This strategy simply is unethical and, furthermore, is hindered by clinical path-

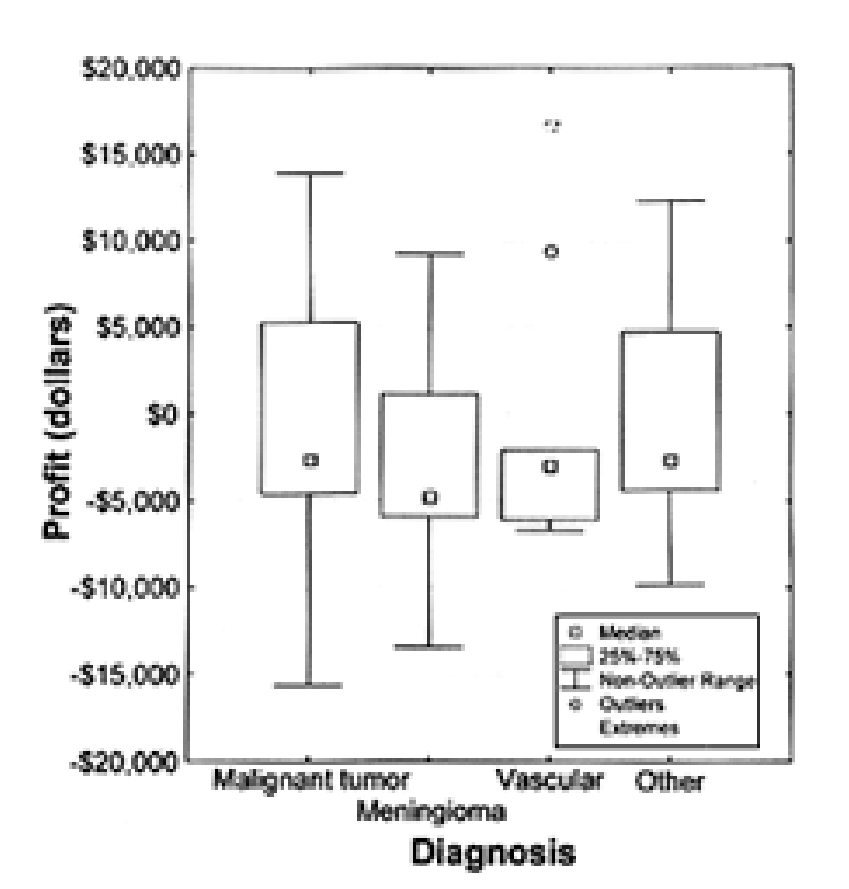

Fig. 3. Box plot of profit categorized by specific patient diagnosis demonstrating that no specific diagnosis tended to influence profit (Kruskal-Wallis test, $\mathrm{p}=0.41$ ). Diagnoses are listed in order of the number of cases with craniotomies for malignant tumor, meningioma, and vascular procedures $(19,18$, and nine cases, respectively). There were 30 craniotomies for other diagnoses.

ways, established clinical LOS criteria, ${ }^{2}$ and representative insurance company-employed case workers who patrol hospitals to determine when a particular patient's "eligibility" for hospitalization has ceased.

\section{Current Study}

The craniotomy clinical pathway was developed at AMC in 1998 by an interdisciplinary team of health care providers, including neurosurgeons, to standardize interventions, decrease variation, and provide a quality-driven plan of care. We continually reviewed our craniotomy pathway to identify areas for improvement. Profitabilityrelated strategies were explored including aspects of efficiency, cost, and utilization. ${ }^{4}$ Ultimately, despite alterations to the pathway, no further cost-saving strategies could be identified. This is exemplified in the present study by the fact that such variables known to influence cost of care, such as operating room and pharmacy costs or individual surgeon profitability, did not influence overall profitability. In fact, room and board, one variable often important in profitability, was shown to be lower in our nonprofitable group. When benchmarking data provided by the University Health System Consortium verified utilization efficiency, targeted LOS goal achievement, and mean cost to be less in our study group than in comparable university medical centers, it was concluded that the clinical pathway was effective and the hospital system efficient in the management of patients undergoing craniotomy. This led to the present analysis to determine why some cases could be managed profitably whereas others with seemingly similar characteristics (operations, LOS, and resource consumption) were nonprofitable. 


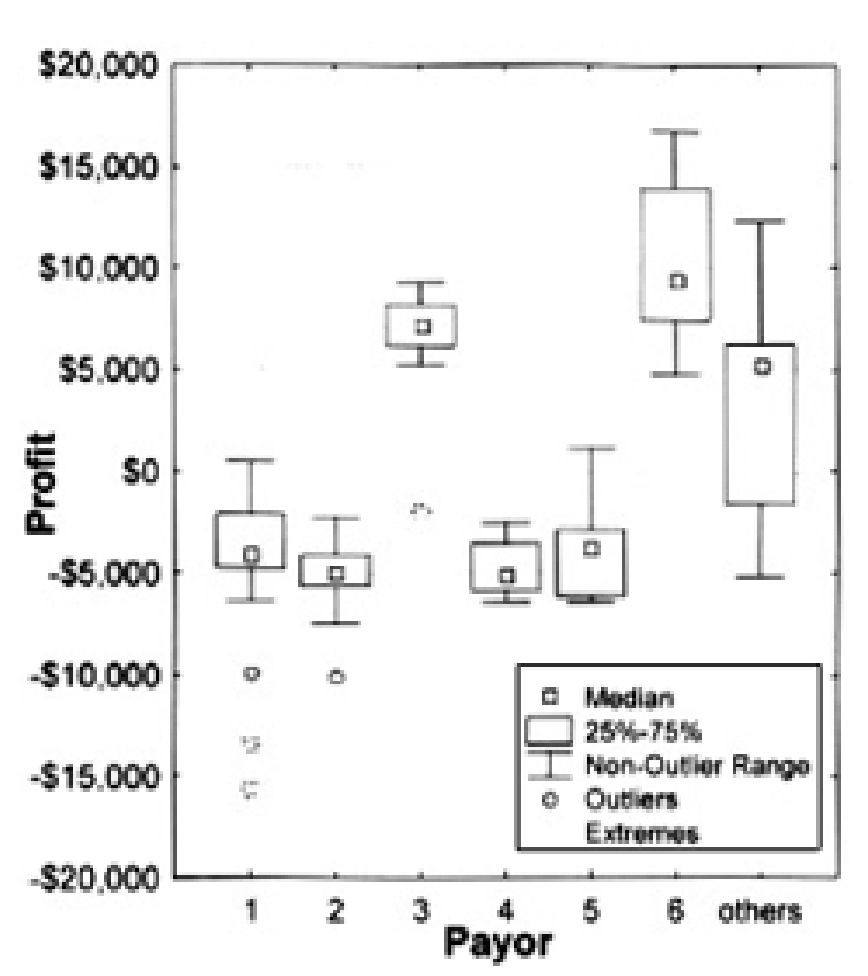

Fig. 4. Box plot of profit categorized by payors demonstrating significant differences in profit (Kruskal-Wallis test, $\mathrm{p}<0.001$ ). Payors are listed in order of the number of cases with craniotomy with Payors 1, 2, 3, 4, 5, and 6 having 26, 14, nine, eight, five, and five cases, respectively. There were nine craniotomies (other) reimbursed by four other payors.

We performed this analysis in an attempt to understand better the effect of expense and revenue on the neurosurgical service by focusing on a single DRG. Diagnosisrelated group 1 was chosen for several reasons: 1) there was a suitable number of patients for analysis; 2) a wellestablished clinical pathway existed to minimize variability for patient management within this DRG; and 3) historically, cases in which craniotomy was performed were perceived to be profitable for the hospital.

It became apparent during the initial analysis that retrieval of data for such a case-by-case analysis was complicated. Our hospital's present information system would require customization if the management information necessary to derive appropriate cost of care-related information was to be readily available for the purpose of contract negotiations. This was particularly important because we found profitability in nearly all PPS cases and lost money on nearly every per diem rate case. In an era of shrinking profit margins, adherence to the traditional wisdom that profits could be by raising volume would have serious consequences if volume were to be increased for procedures that consistently lost money for the hospital.

\section{Importance of Identifying Nonprofitability}

Hospital negotiations involving per diem payors are complex. Generally, a rate for per diem payment is established that covers most of the book of business for the payor. Thus, short of a case-by-case analysis of the cost of delivering care, most negotiations are undertaken using "global" principles and accounting. One option for an isolated DRG payment such as DRG 1 would be as a "carve out" from the contract; this would be accomplished by proving, using databases such as University Health System Consortium data, that the cost of delivering similar care in similar hospitals is higher.

Internal negotiations between physician or clinical services such as neurosurgery and hospital administration are also served by such analyses because programmatic support through capital equipment purchases and program development are facilitated by evidence that patient care is provided efficiently and cost effectively. We believe that the process of cost/revenue analysis, as we have outlined, is a valuable exercise for high-volume procedures and should be part of each hospitals armamentarium for survival.

\section{CONCLUSIONS}

1) Hospital information systems that are designed to capture individual case-related cost of care patients may be helpful in negotiating suitable contracts from per diem payors. 2) The craniotomy-based clinical pathway was successful in mitigating well-known drivers of cost of care such as surgeon variability and resource use between profitable and nonprofitable cases. 3) In today's complex health care environment, attention to each case-related payor reimbursement plan may be as important as having an efficiently run practice.

\section{References}

1. Florin RE: The role of quality in neurosurgical practice: the objective basis of accountability, in Bean JR (ed): Neurosurgery in Transition: The Socioeconomic Transformation of Neurological Surgery. Baltimore: Williams \& Wilkins, 1998, Vol 9, pp 71-95

2. Foulke GE, Schibanoff JM (eds): Inpatient and Surgical Care Optimal Recovery Guidelines. Seattle: Milliman \& Robertson, 1999, pp 5-16

3. Kusske JA: Managed care: the growth of cost containment and the impact on neurosurgical practice, in Bean JR (ed): Neurosurgery in Transition: The Socioeconomic Transformation of Neurological Surgery. Baltimore: Williams \& Wilkins, 1998, Vol 9, pp 3-30

4. Lowe A: Reducing variation in patient care. Nursing responds to capitation. J Nurs Adm 26:14-20, 1996

5. Noetscher CM, Morreale GF: Length of stay reduction: two innovative hospital approaches. J Nurs Care Qual 16:1-14, 2001

6. Roski RA: Changing reimbursement for neurosurgery, in Bean JR (ed): Neurosurgery in Transition: The Socioeconomic Transformation of Neurological Surgery. Baltimore: Williams \& Wilkins, 1998, Vol 9, pp 61-70

7. Waldman JB, McLaughlin MB: Establishing clinical pathways, in Linskey ME, Rutigliano MJ (eds): Quality and Cost in Neurological Surgery. Philadelphia: Lippincott Williams \& Wilkins, 2001, Vol 10, pp 135-151

8. Zehr KJ, Dawson PB, Yang SC, et al: Standardized clinical care pathways for major thoracic cases reduce hospital costs. Ann Thorac Surg 66:914-919, 1998

Manuscript received February 18, 2002.

Accepted in final form March 7, 2002.

Address reprint requests to: A. John Popp, M.D., Department of Surgery, A300B, Albany Medical College, 47 New Scotland Avenue, MC61, Albany, New York 12208. email: poppj@mail.amc.edu. 